\title{
CXCR4/CXCL12/CXCR7 axis is functional in neuroendocrine tumors and signals on mTOR
}

\author{
Luisa Circelli, ${ }^{1, *}$, Concetta Sciammarella ${ }^{2, *}$, Elia Guadagno ${ }^{3}$, Salvatore Tafuto ${ }^{4}$, \\ Marialaura del Basso de Caro ${ }^{3}$, Giovanni Botti ${ }^{1}$, Luciano Pezzullo ${ }^{5}$, Massimo Aria ${ }^{6}$, \\ Valeria Ramundo ${ }^{2}$, Fabiana Tatangelo ${ }^{7}$, Nunzia Simona Losito $^{7}$, Caterina Ieranò ${ }^{1}$, \\ Crescenzo D'Alterio ${ }^{1}$, Francesco Izzo $^{8}$, Gennaro Ciliberto ${ }^{9}$, Annamaria Colao ${ }^{2}$, \\ Antongiulio Faggiano $^{5}$, Stefania Scala ${ }^{1}$

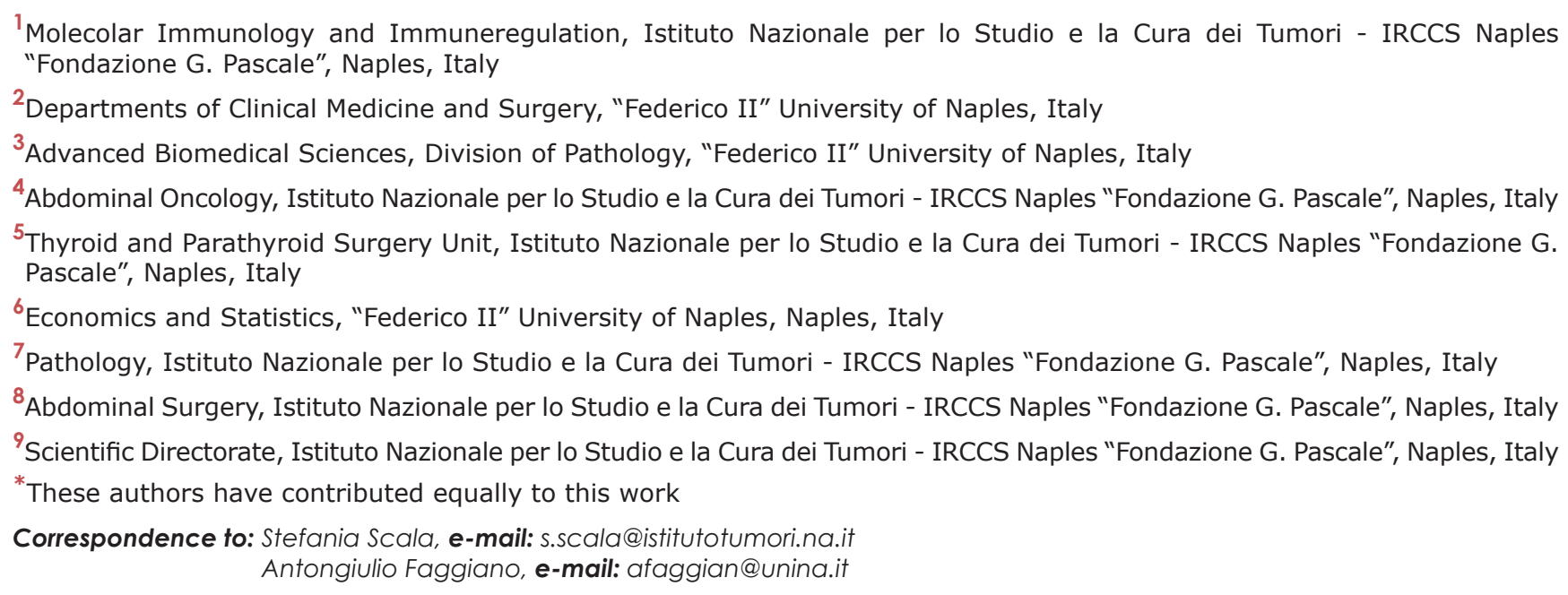
Keywords: chemokine, mTOR, NET, clinical outcome, grading

Received: September 18,2015 Accepted: January 06, $2016 \quad$ Published: February 26, 2016

\section{ABSTRACT}

Objective: To evaluate the possible crosstalk between $\mathrm{C}-\mathrm{X}-\mathrm{C}$ chemokine receptor 4 (CXCR4)/C-X-C motif chemokine 12 (CXCL12)/C-X-C chemokine receptor 7 (CXCR7) axis with the mammalian target of rapamycin (mTOR) pathway in neuroendocrine tumors (NETs).

Methods: Sixty-one human NETs were included into the study. CXCR4/CXCL12/ CXCR7 axis and mTOR pathway were assessed by QRT-PCR and immunohistochemistry (IHC). The effect of mTOR inhibitor, RAD001, was evaluated on CXCR4 pathway through proliferation and p-Erk and p-AKT induction. Results: CXCR4/CXCL12/ CXCR7 axis and p-mTOR were found to be active and correlated with grading, Ki67 index and tumor stage. mTOR pathway activation significantly correlated with poor prognosis. In human NET cells, CXCL12 induced mTOR signalling while AMD3100 (CXCR4-antagonist) impaired it. The mTOR-antagonist, RAD001, impaired the CXCL12dependent induction of CXCR4 downstream effectors. Combination of AMD3100 and RAD001 potentiate cell growth inhibition.

Conclusions: CXCR4/CXCL12/CXCR7 axis is active in NETs and signals on mTOR. CXCR4 might be considered a prognostic factor in NETs. Combined treatment with AMD3100 and RAD001 may provide clinical benefits in NET patients with drugresistant. 


\section{INTRODUCTION}

Neuroendocrine tumors are relatively rare (incidence about 1-5 cases/100,000/year) and heterogeneous neoplasms with increasing incidence and prevalence [1]. NETs display variegate biological and clinical course in which primary site, stage and grading affect prognosis. According to the last classification of the World Health Organization (WHO) 2010, gastroenteropancreatic NETs (GEP-NETs) are classified into low (G1) intermediate (G2) and high-grade (G3) lesions, based on mitotic count and Ki67 score [2, 3]. High grade lesions (neuroendocrine carcinomas, NEC) present a mitotic count of $>20$ for 10 high-powered fields (HPF) or a Ki67 proliferation index of $>20 \%$ [4], and are generally treated with platinumbased chemotherapy regimens. On the contrary, welldifferentiated, low- and intermediate-grade NETs (G1, G2) are clinically indolent with a low proliferation index and good prognosis. Approximately $50 \%$ of NETs present with metastases at the diagnosis; the conventional treatment is based on biological therapies (somatostatin analogues, $\alpha 2 b$-interferon) or chemotherapy. Nevertheless no significant impact on survival has been obtained [5].

Medullary thyroid carcinoma (MTC) is a malignant tumor of the thyroid (5 to $10 \%$ of all thyroid malignancies) originating in C-cell, deriving from the neural crest, expressing neuroendocrine markers (chromogranine and synaptofisin). Stage in thyroid cancer is based on TNM Classification and separate stage groupings are recommended for different histotypes [6,7]. MTC is a well differentiated NET, slowly growing and insensitive to chemotherapy. Somatostatin analogues and interferon only ameliorates symptoms, while the newly identified TK-inhibitors vandetanib and cabozantinib have antiproliferative effect [8-11].

As of today mammalian target of rapamycin (mTOR) inhibitors, such as RAD001, have been approved as second-line therapy in patients with progressive pancreactic NETs [12-14]. mTOR is a serine-threonine kinase regulating different cellular processes, such as metabolism, nutrient sensing, translation and cell growth [15]. Both mTOR molecular complexes, mTORC1 and mTORC2, use mTOR as the catalytic subunit. mTORC1 increases cell growth and proliferation through ribosomal protein S6 kinase 1 (S6K1) and eukaryotic translation initiation factor 4E (eIF4E)-binding protein (4EBP1) $[16,17]$. mTORC2 phosphorylates Protein kinase B (Akt), at Thr450 and Ser473 [18, 19]. Evidence suggest that the phosphatidylinositol 3-kinase (PI3K)/Akt/ mTOR signaling is involved in NET tumorigenesis and progression, given its critical role in cell proliferation and angiogenesis. Tamburrino et al. showed that the PI3K/ Akt/mTOR pathway is crucial to MTC tumorigenesis [20] and in a recent review, Manfredi et al. showed that MTC cell proliferation depends on PI3K/Akt/mTOR signaling, suggesting a novel therapeutic target for MTC [21]. A potent anti-proliferative effect of RAD001, mediated by the cell cycle arrest in G0/G1 phase, but not apoptosis, was recently shown in MTC cells [18]. To date, mTOR inhibitors failed to provide relevant benefits and patients often develop resistance to therapy and progression of disease [22]. Thus there are still unmet need for new therapeutic targets [23-25].

The chemokine CXCL12 binds the two receptors CXCR4 and CXCR7 and transduces on the mTOR pathway in pancreatic cancer, gastric cancer, $\mathrm{T}$ cell leukemia cell and in human renal cancer cells [2628]. Chemokines are a superfamily of chemoattractant proteins that induce cytoskeletal rearrangement, firm adhesion to specific cells and directional migration [29]. The binding of chemokines to their receptors triggers a cascade of events, including receptor dimerization, recruitment of heterotrimeric $\mathrm{G}$ proteins, and activation of the Janus kinase and Signal Transducer and Activator of Transcription (STAT), PI3K and mitogen-activated protein kinases (MAPK), extracellular signal-regulated kinases (ERK) pathways. Emerging evidence indicates that chemokine receptors pathways control tumor development, including tumor growth, progression, and metastasis $[30,31]$ and that CXCL12/CXCR4 activation induces migration and metastatic processes [32-34].

The aim of the study was to evaluate the CXCR4/ CXCL12/CXCR7 axis and the possible crosstalk with mTOR pathway in NETs to identify new prognostic and therapeutic targets.

\section{RESULTS}

\section{CXCR4/CXCL12/CXCR7 pathway is overexpressed in GEP-NETs and MTCs}

Clinical and pathological characteristics of the study population are summarized in Table 1. CXCR4, CXCL12 and CXCR7 mRNA expression was evaluated in 61 NETs and in 36 normal tissues, as shown in Figure 1. CXCR4, CXCL12 and CXCR7 were significantly overexpressed in NETs as compared to normal samples $(\mathrm{p}<0.0001)$.

CXCR4, CXCL12 and CXCR7 protein levels were evaluated by IHC in 53/61 patients (31 GEP-NETs, 18 MTCs and 4 unknown). A positive immunostaining was found in 28/31 (90\%) GEP-NETs and 13/18 (72\%) MTCs for CXCR4, in 29/31 (93\%) GEP-NETs and 17/18 (94\%) MTCs for CXCR7 and in 30/31 (97\%) GEP-NETs and 16/18 (89\%) MTCs for CXCL12 (Table 2) (Figure 2).

A significant correlation was identified among CXCR4, CXCR7 and CXCL12 (Table 3). In GEP-NETs, a significant positive correlation was observed between the CXCR4/CXCL12/CXCR7 immunostaining score and Ki67 score $(p<0.05)$. CXCR4 and CXCR7 were more expressed in $\mathrm{G} 3$ than in $\mathrm{G} 1 / \mathrm{G} 2(\mathrm{p}<0.05)$. In MTCs, CXCR7 and CXCL12 immunostaining was significantly higher in stage II-III as compared to stage I $(p<0.05)$. 
Table 1: Clinicopathologic characteristics of patients with gastroenteropancreatic NETs and Medullary thyroid carcinoma

\begin{tabular}{lc}
\hline Characteristics & Numbers \\
\hline no. of patients & 61 \\
Median age in years (range) & $64.5(28-86)$ \\
Gender (Males/Females) & $27 / 34$ \\
Primary tumor & 4 \\
Unknown & 21 \\
Thyroid & 7 \\
Colon & 2 \\
Appendix & 2 \\
Duodenum & 14 \\
Pancreas & 1 \\
Stomach & 10 \\
Ileum & \\
pTNM of MTC & 8 \\
Stage I (T1N0M0) & 9 \\
Stage II (T2-T3N0M0) & 4 \\
Stage III (T1-T3N1M0) & 0 \\
Stage IV & \\
Tumor grading of GEP-NETs and unknown & 11 \\
G1 & 14 \\
G3 & 15 \\
\hline
\end{tabular}
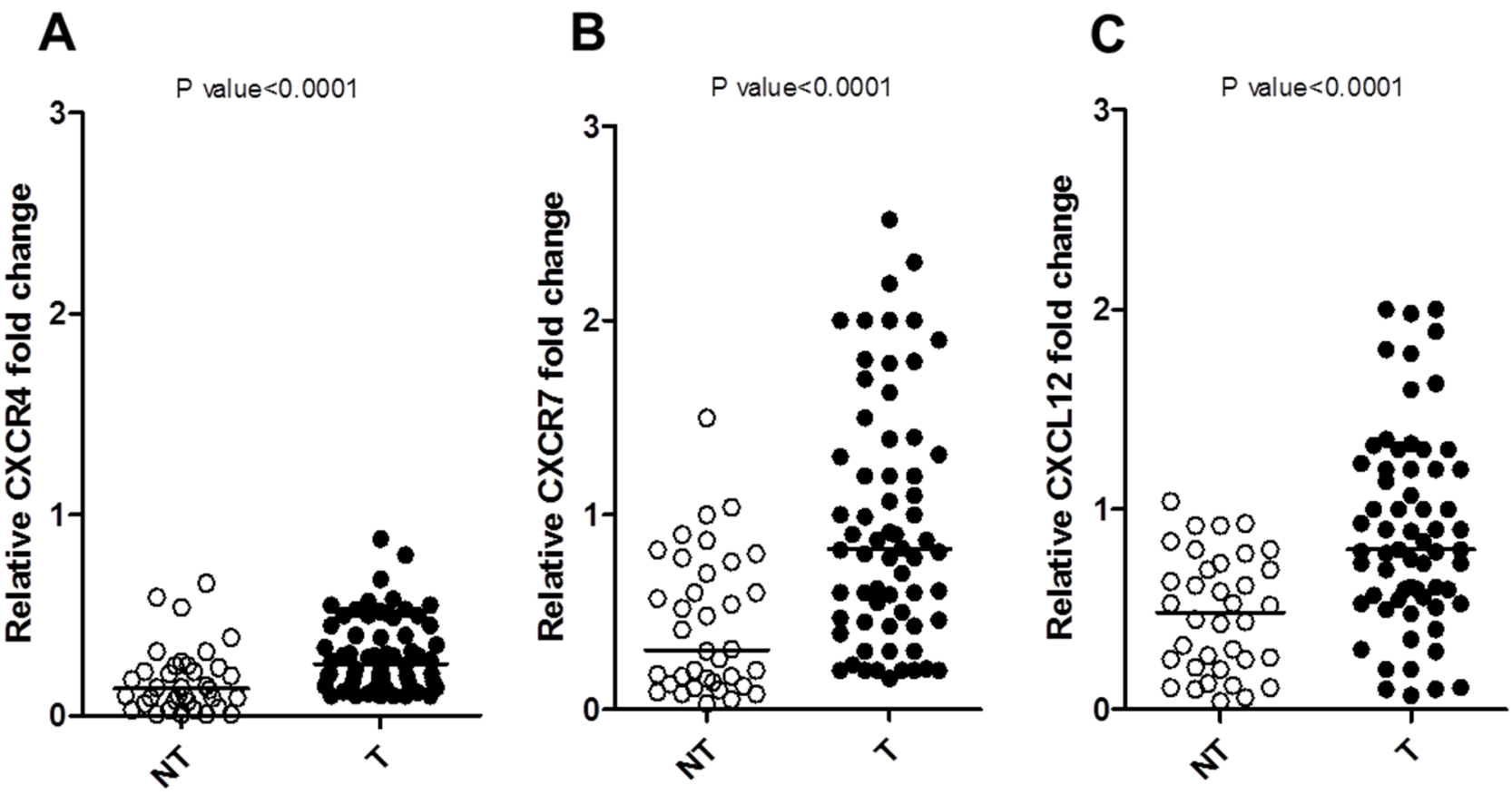

Figure 1: Relative mRNA expression of NETs (T) versus normal tissue (NT) by real-time RT-PCR. A. CXCR4, B. CXCR7 and C. CXCL12 expression levels of T (Y axis) were calculated relative to the mean level of NT. All experiments have been performed in duplicate, and the average value of the results was plotted on the diagram. P value was calculated with Mann-Whitney test, two-tailed. 
Neither in GEP-NETs nor in MTCs, CXCR4/CXCL12/ CXCR7 level correlated with clinical outcome.

The mTOR pathway was evaluated; 18 of 31 GEPNETs (58\%) were positive for both mTOR and p-mTOR, $10(56 \%)$ and $14(78 \%)$ of 18 MTCs were positive for mTOR and p-mTOR respectively. Moreover, 19/31 (61\%) GEP-NETs and 12/18 (67\%) MTCs were positive for p-S6K1, 16/31 (52\%) GEP-NETs and 9/18 (50\%)
MTCs for p-4EBP1 (Table 2). As expected, a significant positive correlation was observed between mTOR and $\mathrm{p}$-mTOR $(\mathrm{p}<0.05)$, mTOR and both $\mathrm{p}-\mathrm{S} 6 \mathrm{~K} 1$ and $\mathrm{p}-4 \mathrm{EBP} 1$ $(p<0.05$ and $p<0.0001)$ and between $p$-mTOR and its two effectors $(\mathrm{p}<0.05)$ (Table 3$)$. The immunostaining score for $\mathrm{p}$-mTOR $(\mathrm{p}<0.05)$ was significantly higher in G1/G2 tumors than in G3 in GEP-NETs, while it was not significantly correlated to tumor stage in MTCs.

\section{Table 2: Immunostaining of CXC and mTOR factors in gastroenteropancreatic NETs and}

Medullary thyroid carcinoma

\begin{tabular}{lcc}
\hline & GEP-NETs & \multicolumn{2}{c}{ MTCs } \\
\cline { 2 - 3 } Protein & Positive \% & Positive \% \\
\hline CXCR4 & 90 & 72 \\
CXCR7 & 93 & 94 \\
CXCL12 & 97 & 89 \\
mTOR & 58 & 56 \\
p-mTOR & 58 & 78 \\
p-S6K1 & 61 & 67 \\
p-4EBP1 & 52 & 50 \\
\hline
\end{tabular}

A

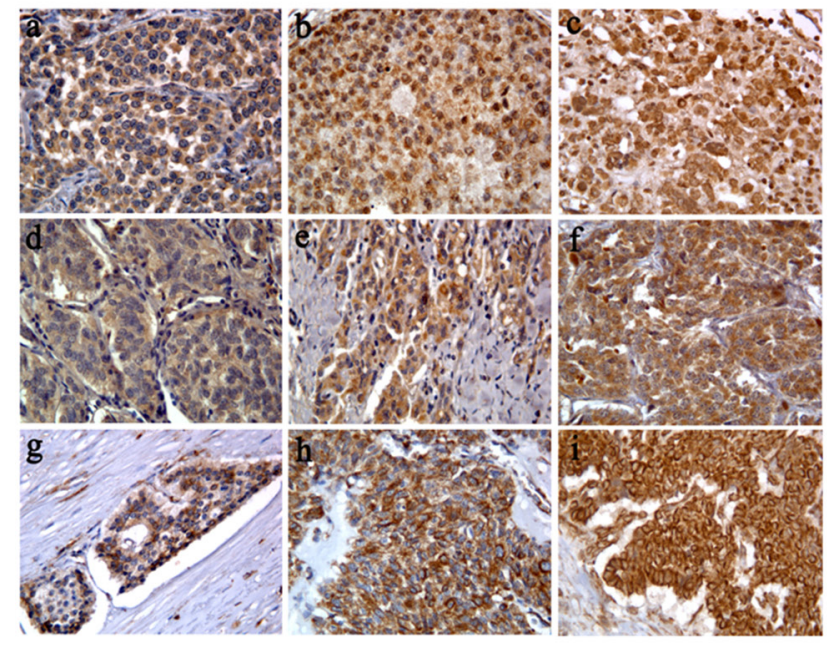

B

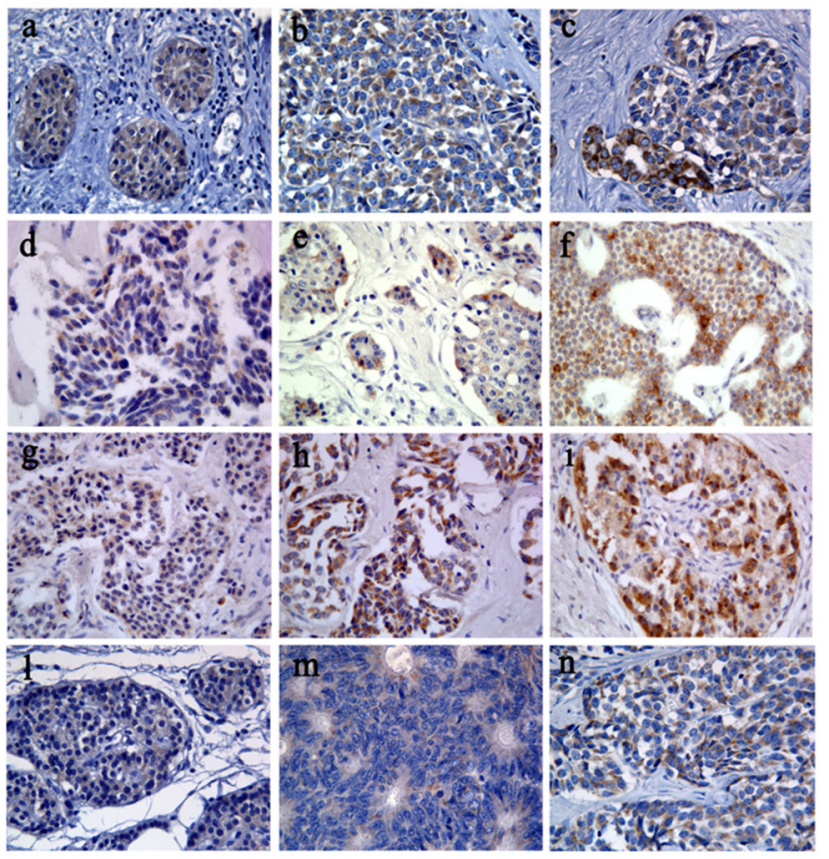

Figure 2: CXCR4/CXCL12/CXCR7 and mTOR pathway expressions in neuroendocrine tumor patients. A. CXC immunostaining. NEC G3 stomach: weak CXCR4 (a), NET G2 ileum: moderate CXCR4 (b), NEC G3 colon: strong CXCR4 (c); NET G1 ileum: weak CXCR7 (d), NET G2 pancreas: moderate CXCR7 (e), NET G2 pancreas: strong CXCR7 (f); NET G1 ileum: weak CXCL12 (g), moderate CXCL12 (h), NEC G3 colon: strong CXCL12 (i) (original magnification, 400×). B. mTOR immunostaining. NET G1 appendix: weak mTOR (a), NEC G3 gastric: moderate mTOR (b), NEC G3 pancreas: strong mTOR (c); NET G1 pancreas: weak p-mTOR (d), NET G1 ileum: moderate p-mTOR (e), NET G1 ileum: strong p-mTOR (f); NETG2 ileum: weak p-S6K1 (g), NEC G3 colon: moderate p-S6K1 (h), NEC G3 pancreas: strong p-S6K1 (i); NET G1 appendix: weak p-4EBP1 (1), NET G2 colon: moderate p-4EBP1 (m), NEC G3 gastric: strong p-4EBP1 (n) (original magnification, 400×). 
In the whole NET population, $\mathrm{p}$-mTOR $(\mathrm{p}<0.01)$ and $\mathrm{p}-4 \mathrm{EBP} 1 \quad(\mathrm{p}<0.01)$ scores were significantly high in patients with unfavorable outcome. In particular in GEPNETs, p-mTOR $(p<0.05)$, p-S6K1 $(p<0.05)$ and p-4EBP1 $(p<0.05)$ scores were high in patients with unfavorable outcome. In MTCs, there was no significant correlation between mTOR pathway immunostaining and outcome.

Finally, CXCR4 significantly correlated with mTOR $(\mathrm{p}<0.01)$ and $\mathrm{p}-\mathrm{S} 6 \mathrm{~K} 1(\mathrm{p}<0.05)$ while CXCR7 with p-S6K1 $(\mathrm{p}<0.05)$ (Table 3$)$.

\section{CXCR4/CXCL12/CXCR7 axis signals on mTOR in NET cell lines}

To evaluate the CXCR4/CXCL12/CXCR7 axis in NET cell lines, NCI-H727 (bronchial-NET) and BON
(pancreatic-NET) were tested for CXCL12 induced signaling. As shown in Figure 3, in BON and NCI-H727, CXCR4 and CXCR7 were overexpressed. CXCL12 induced the mTOR targets p-S6K1 and p-4EBP1 in BON cells and the CXCL12-induction was inhibited by the specific CXCR4 antagonist, AMD3100 (Figure 4). As expected, addition of mTOR inhibitor, RAD001, blocked the downstream mTOR effectors (Figure 4A); RAD001 modestly impaired CXCL12-mediated p-Erk1/2, p-P38 and p-Akt induction. Moreover CXCL12 induced the phosphorylation of Erk1/2 and P38, which was inhibited by AMD3100 (Figure 4B).

Then, the effects of CXCL12 were evaluated on the BON and NCI-H727 cells growth. AMD3100 and RAD001 reduced cell growth already at 24 hrs (Figure 5). Moreover,

Table 3: Spearman correlation of CXC and mTOR factors in gastroenteropancreatic NETs and Medullary thyroid carcinoma

\begin{tabular}{lrrrrrr}
\hline & CXCR7 & CXCL12 & mTOR & p-mTOR & p-S6K1 & p-4EBP1 \\
\hline CXCR4 & $\boldsymbol{\rho}=\mathbf{0 . 4 1 5}$ & $\boldsymbol{\rho}=\mathbf{0 . 2 9 8}$ & $\boldsymbol{\rho}=\mathbf{0 . 3 9 5}$ & $\rho=0.081$ & $\boldsymbol{\rho}=\mathbf{0 . 3 1 9}$ & $\rho=0.103$ \\
$P$ & $\mathbf{0 . 0 0 2}$ & $\mathbf{0 . 0 3 2}$ & $\mathbf{0 . 0 0 3}$ & 0.564 & $\mathbf{0 . 0 2 0}$ & 0.481 \\
CXCR7 & & $\boldsymbol{\rho}=\mathbf{0 . 4 1 8}$ & $\rho=0.099$ & $\rho=-0.053$ & $\boldsymbol{\rho}=\mathbf{0 . 2 8 5}$ & $\rho=0.139$ \\
$P$ & & $\mathbf{0 . 0 0 2}$ & 0.486 & 0.711 & $\mathbf{0 . 0 4 0}$ & 0.347 \\
CXCL12 & & $\rho=0.248$ & $\rho=-0.216$ & $\rho=0.070$ & $\rho=0.231$ \\
$P$ & & 0.077 & 0.124 & 0.624 & 0.114 \\
mTOR & & & $\boldsymbol{\rho}=\mathbf{0 . 2 9 9}$ & $\boldsymbol{\rho}=\mathbf{0 . 3 3 5}$ & $\boldsymbol{\rho}=\mathbf{0 . 5 7 8}$ \\
$P$ & & & $\mathbf{0 . 0 2 9}$ & $\mathbf{0 . 0 1 4}$ & $<\mathbf{0 . 0 0 0 1}$ \\
p-mTOR & & & & $\boldsymbol{\rho}=\mathbf{0 . 3 5 2}$ & $\boldsymbol{\rho}=\mathbf{0 . 3 2 6}$ \\
$P$ & & & & $\mathbf{0 . 0 1 0}$ & $\mathbf{0 . 0 2 2}$ \\
p-S6K1 & & & & & $\boldsymbol{\rho}=\mathbf{0 . 3 1 2}$ \\
$P$ & & & & & & $\mathbf{0 . 0 2 9}$ \\
\hline
\end{tabular}

A

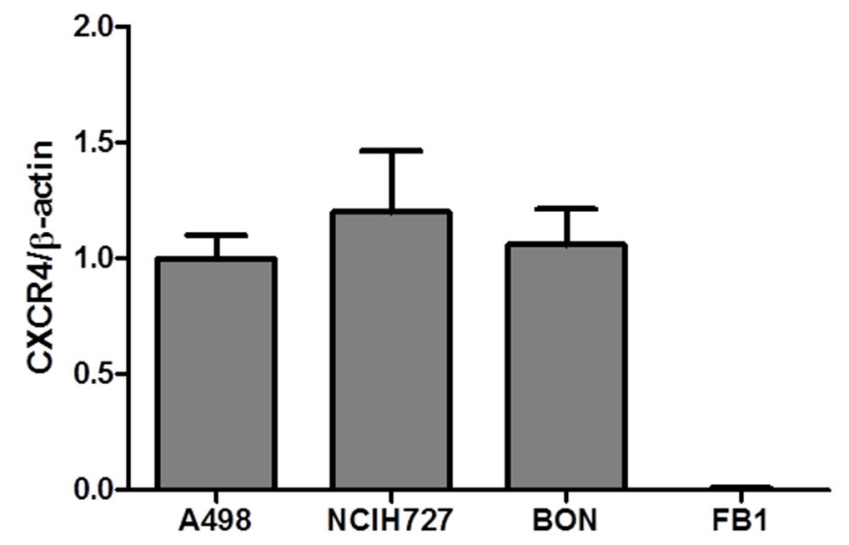

B

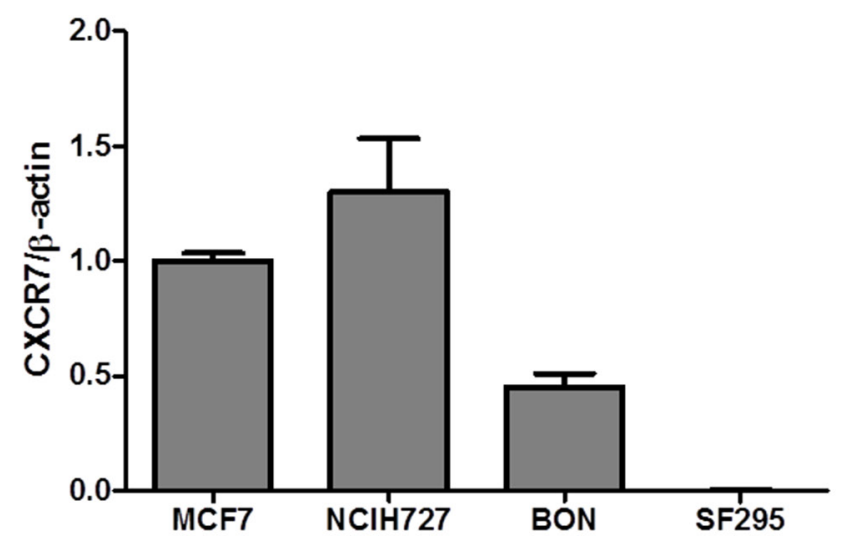

Figure 3: Relative mRNA expression in cell lines. A. CXCR4 and B. CXCR7 mRNA expression was analyzed in NCI-H727 and BON through RT-PCR. A498 and FB1, human renal cancer and a human anaplastic thyroid cancer cell lines, as positive and negative control for CXCR4 respectively. MCF 7 and SF295, breast cancer cell line and a human glioblastoma, as positive and negative control for CXCR7 respectively. 


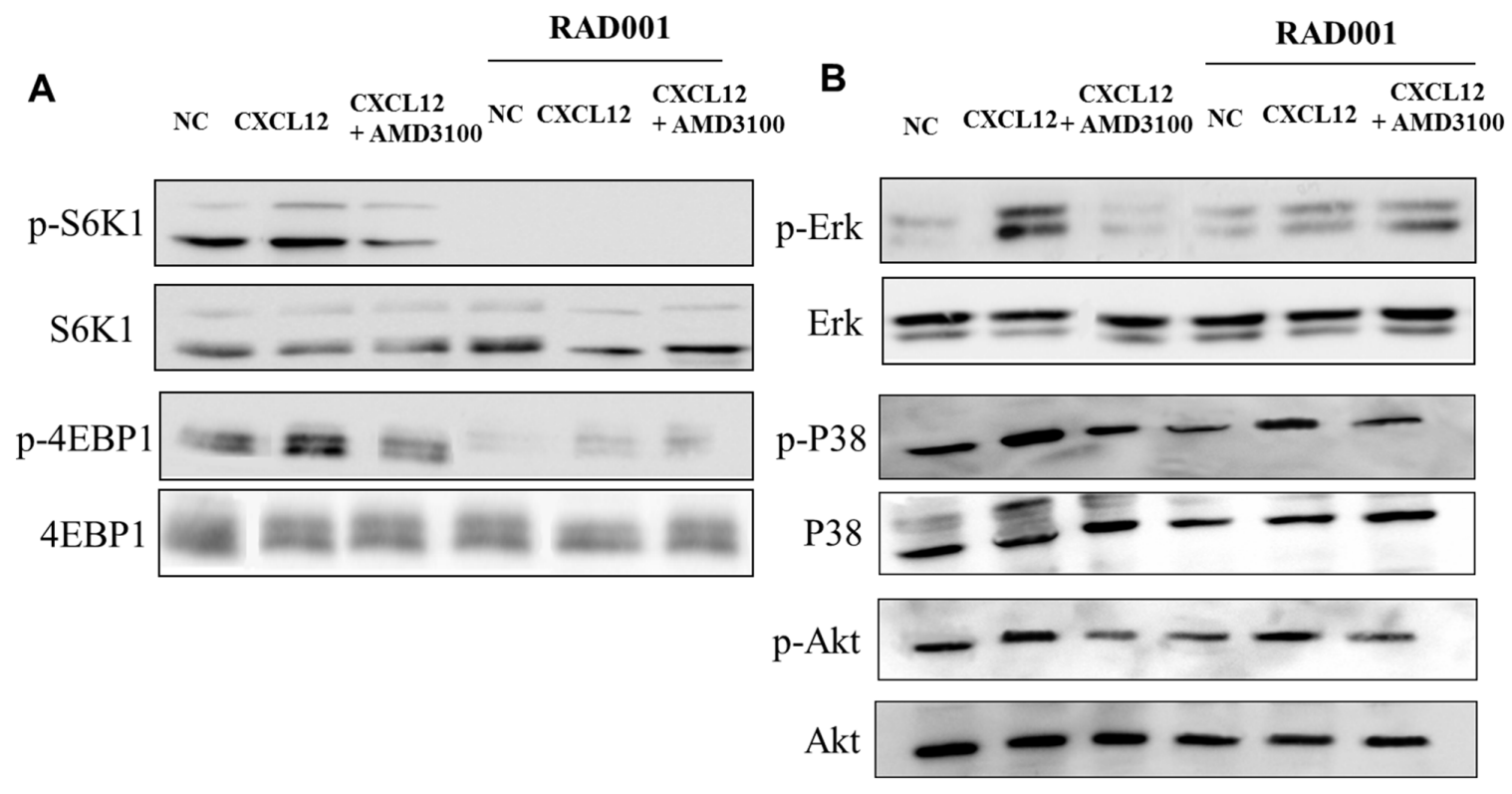

Figure 4: CXCR4 signals through the mTOR signaling pathway in BON cell line. A. BON were stimulated with CXCL12 $(100 \mathrm{ng} / \mathrm{ml})$ for $10 \mathrm{~min}$ and after with AMD3100 $(5 \mu \mathrm{M})$, with or without the RAD001 $(1 \mu \mathrm{M})$; p-S6K1 and p-4EBP1 protein expressions were measured using western blot analysis. Representative data from one of three experiments. B. p-Erk1/2, p-P38 and p-Akt activation was measured in the same conditions. Total Erk1/2, P38 and Akt proteins were used for normalization. Representative data from one of three experiments. NC: Normal control
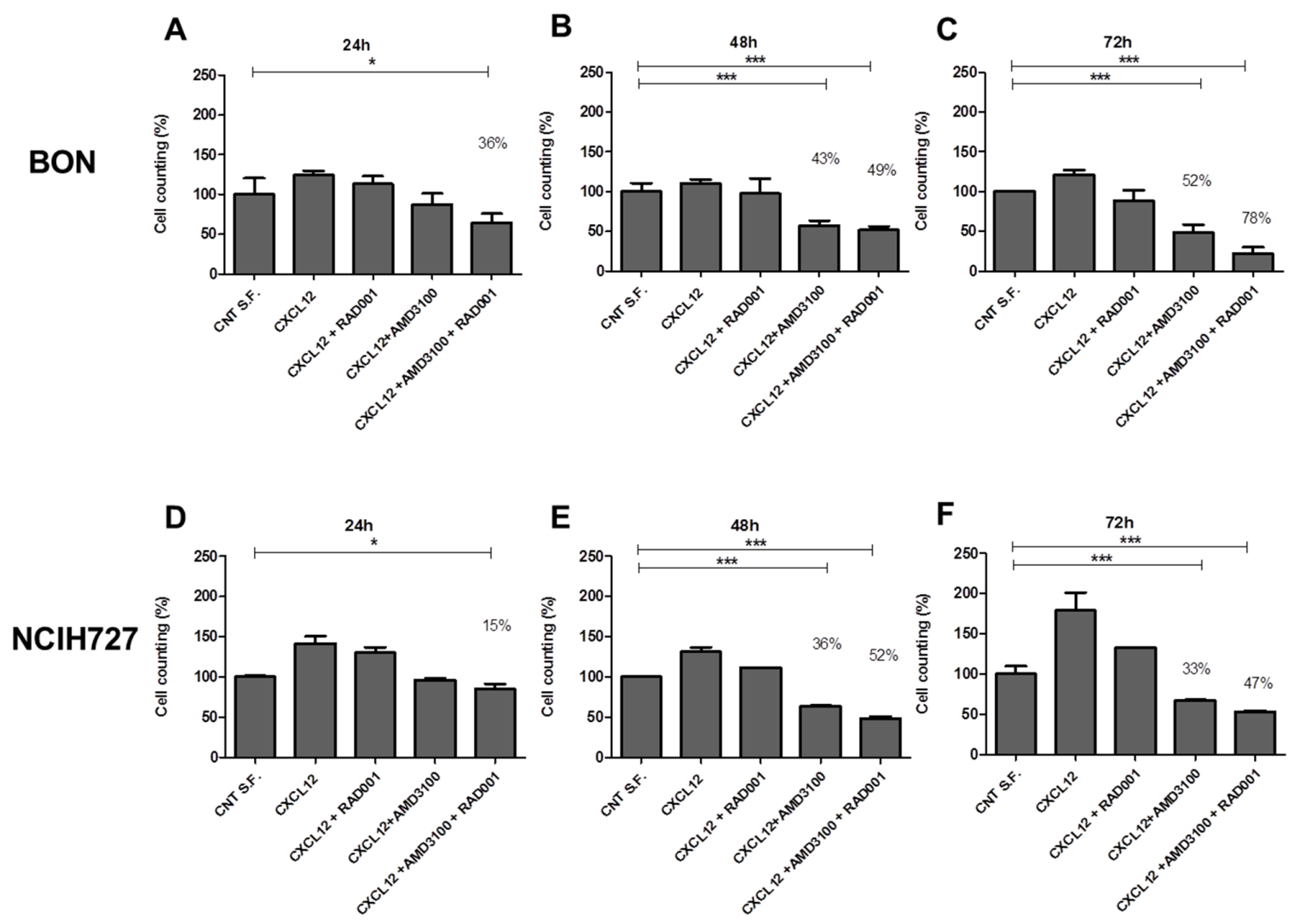

Figure 5: Effect of RAD001 and AMD3100 on cells proliferation. BON after $24 \mathrm{hrs}$ A., $48 \mathrm{hrs}$ B. and $72 \mathrm{hrs}$ C.; NCI-H727 after 24hrs D., 48hrs E. and 72hrs F. in presence of CXCL12 (100 ng/ml) and AMD3100 (5 mM), with or without of RAD001 (1 $\mu$ M). Results are representative of three different experiments performed. Each column represents the mean \pm S.D. Statistical significances were calculated by One-way ANOVA with Bonferroni correction. ${ }^{*} \mathrm{P}<0.5, * * \mathrm{P}<0.01, * * * \mathrm{P}<0.001$. Control with serum free (CNT S.F.) versus treatments. 
the addition of AMD3100 significantly $(\mathrm{p}<0.05)$ enhanced RAD001 anticancer activity after 24 hrs (Figure 5).

\section{DISCUSSION}

To shed further insight on NET biology the role of the CXCR4/CXCL12/CXCR7 axis and the possible interconnection with the signaling on mTOR pathway was investigated. It was observed that CXCR4, CXCL12 and CXCR7 increased in both GEP-NETs and MTCs. Recent evidence demonstrated that CXCR4 is expressed in lung NETs [35] and significantly correlated with negative patient outcome [36]. To the best of our knowledge this is the first time that the whole CXCR4/CXCL12/CXCR7 axis was found overexpressed in NETs and correlating with tumor grading and Ki67 [37]. In MTCs, high CXCR7 and CXCL12 expression was detected in more advanced tumors and high p-mTOR level was observed in G1/G2 GEP-NETs compared to G3. The correlation between tumor grading and activation of p-mTOR in NETs is controversial $[38,39]$ probably because NETs are rare tumors but also because the poor differentiated NETs are underrepresented in the most of studies. Furthermore these data confirmed that the mTOR pathway is broadly expressed in NETs and seems prognostic factors [40]. Crosstalks between CXCR4, CXCL12 and PI3K/mTOR had been previously described in peritoneal disseminated gastric cancer [26], pancreatic cancer [41], human T-cel leukemia [42] and renal cancer [28]. The existence of an interplay between CXCR4, CXCL12 and PI3K/ mTOR has been demonstrated in NETs, where CXCL12 induced p-S6K1 and p-4EBP1 in NET cell lines and the CXCL12-induction was inhibited by AMD3100. The addition of RAD001 blocked and further reduced the mTOR downstream effectors. In addition, at the immunohistochemical analysis, CXCR4 correlated with mTOR and p-S6K1 and CXCR7 with p-S6K1. As RAD001 impaired CXCL12-mediated p-Erk1/2 and p-P38 induction, the conclusion was that mTOR inhibition is downstream of CXCR4 axis.

CXCL12 dependent CXCR4 signals on mTOR inducing human NET cell growth while the inhibitors AMD3100 and RAD001 impaired cell growth with an additive effect. We recently developed a new class of cyclic peptidic CXCR4 antagonists [43], that could be evaluated in combination with RAD001 in NET patients. The goal of this study will be to provide a dose and regimen for the combined treatment and its tolerability, as reported by other ongoing clinical trials exploiting the interaction of mTOR with other pathways [44].

In conclusion, to the best of our knowledge, this is the first report of CXCR4 expression and signaling in NETs through the mTOR pathway. CXCR4 may represent a new prognostic factor in NET tumors and mTOR inhibitors coupled with the CXCR4 inhibitors could represent a novel pharmacological approach to prevent mTOR drug resistance.

\section{MATERIALS AND METHODS}

\section{Patients and specimens}

Sixty-one patients diagnosed with NETs and followed in two Units of the Multidisciplinary NET group of Naples (the "Federico II" University Hospital and the Istituto Nazionale per lo Studio e la Cura dei Tumori, Fondazione " G. Pascale" of Naples) were enrolled. Out of 61 patients, 53 tumor samples were available as paraffin embedded tissue (31 GEP-NETs, 4 unknown primary origin and 18 MTCs) and 8 as fresh tissue (5 GEP-NETs and 3 MTCs). GEP-NETs were classified according to the WHO International Histological Classification of Endocrine Tumors 2010 [37, 45] while MTCs were classified following the latest staging system (TNM) valid for thyroid tumors [7]. Normal tissue samples (7 thyroid, 29 gastrointestinal) were available for comparative studies.

Expression analysis by quantitative real-time PCR was performed on tumor samples of the whole NET population (53 paraffin embedded and 8 fresh tissues) and 36 normal tissue samples (28 paraffin embedded and 8 fresh tissues) while immunohistochemistry was performed on 53 tumors. Clinical course of disease after surgery was classified as favourable (no evidence of disease, no. 23) and unfavourable (disease persistence no. 11, death for tumor progression no. 10). Follow-up data were not available in 17 patients.

The study was approved by the institutional review board of the Institutions involved in the study (protocol $n^{\circ} 102 / 2013$ approved by the Federico II University Ethical Committee). Informed consent was obtained from patients for their tissues to be used in research.

The data were analyzed anonymously and all clinical investigation have been conducted according to the principles expressed in the Declaration of Helsinki.

\section{Immunohistochemistry}

Immunohistochemistry (IHC) was performed using the primary monoclonal antibodies: CXCR4, CXCR7 and CXCL12 were from R\&D Systems; mTOR, p-mTOR (Ser2448) and p-S6K1 (Thr389) were from Cell Signaling Technology; p-4EBP1 (Thr70) was from Abcam. The specificity of all reactions was validated in parallel with negative controls obtained omitting primary antibodies. Immunohistochemical findings were independently evaluated by four pathologists (EG, MDBDC, SL, FT). Cases with conflicting scores were reviewed jointly with a multi-head microscope until a consensus was reached. All cases were evaluated using a semi-quantitative scoring (IRS) system [46]. Staining intensity was scored as 0 (negative), 1 (weak), 2 (moderate), or 3 (strong) 
(Figure 1). The percentage of tumor cells stained were scored as 0 (none), 1 (1-10\%), 2 (11-50\%), 3 (51-80\%), $4(>80 \%)$. The intensity and percentage were multiplied, resulting in an individual immunoreactivity score (IRS) ranging from 0 to 12 . The raw expression scores were used for correlation analysis. For correlation with clinic pathological variables, any expression of CXCR4, CXCR7, CXCL12, mTOR, p-mTOR, p-S6K1 and p-4EBP1 (IRS 1-12) was considered positive.

Ki67 score was evaluated in GEP-NETs tumor samples by counting $>2000$ cells in immunohistochemical samples representative of the tumour, and a percentage of immunoreactivity (Labeling Index) was calculated [47]. The mitotic count was evaluated according to the method recommended in the 7 th edition of the AJCC Cancer Staging Manual: enumerate mitoses in the most mitotically active area ("hot spot") and then extend mitotic count to adjacent contiguous fields. If no mitotic activity was evident, random representative tumor fields were scanned for mitoses.

\section{Cell culture}

Human NET cell lines used in this study include a Bronchial-NET cell line (NCI-H727) and a pancreaticNET cell line (BON). NCI-H727 cell line was obtained from American Type Culture Collection (ATCC) and BON cell line was gifted by Professor Papotti (University of Turin at San Luigi Hospital, Orbassano).

NCI-H727 was maintained in Rosswell Park Memorial Institute (RPMI)-1640 and BON was cultured in Dulbecco's Modified Eagle Medium:Nutrient Mixture F-12 (DMEM/F12) both supplemented with 10\% fetal bovine serum (FBS), 2 nM L-glutamine, penicillin 10, 000 $\mathrm{IU} / \mathrm{mL}$ and streptomycin 10, $000 \mu \mathrm{g} / \mathrm{mL}$ (all from SigmaAldrich) in a humidified atmosphere containing 5\% CO2 at $37^{\circ} \mathrm{C}$. Human renal cancer cells (A498), human breast adenocarcinoma cells (MCF-7) and human glioblastoma cells (SF295) were obtained from the NCI-Drug Screen Program and human anaplastic thyroid cancer cells (FB1) were obtained from the courtesy of Prof. Rosamarina Melillo, Federico II University, Naples-Italy.

\section{RNA extraction and quantitative real-time PCR}

RNA was isolated from NETs fresh tissue or paraffin-embedded tissues using the RNeasy Mini kit (Qiagen, Hilden, Germany) and RNeasy FFPE Kit according to manufacturer's instructions. RNA was synthesized for cDNA using SuperScript III Reverse Transciptase (Invitrogen). Quantitative real-time PCR was performed using SYBR Green PCR Master Mix (Applied Biosystems, Foster City, CA). $\beta$-actin gene was used as an endogenous control for sample normalization. Data were collected and quantitatively analyzed on an ABI Prism 7000 System (Applied Biosystems).
The gene specific primers used for amplification were as follows:

CXCR4: Fw 5'-TGGGTGGTTGTGTTCCAGTTT-3'

Rw 5'-ATGCAATACCAGGACAGGATGA-3', CXCR7: Fw 5'-GATTGCCCGCCTCAGAAC-3'

Rw 5'-GCAGGACGCTTTTGTTGG-3', CXCL12: Fw 5'-TGTGGCACTCAGATACCGACT-3' Rw 5'-CCCACAGAGCCAATCACT-3'.

A498 and MCF7 cell lines were used as CXCR4 and CXCR7 positive control, respectively; FB1 and SF295 as CXCR4 and CXCR7 negative control, respectively. Samples were run in triplicate, and their relative expression was calculated in the following formula using $\beta$-actin as endogenous control: $2^{-\Delta \Delta \mathrm{Ct}}$.

\section{Western blot}

BON cell line was allowed to grow in T75 flasks until 70\% confluence. The CXCR4 inhibitor, AMD3100, was obtained from Sigma (St. Louis, MO, USA) and RAD001 from Novartis. Complete media was replaced with CXCL12 (100 ng/ml) for $10 \mathrm{~min}$ and after with AMD3100 $(5 \mu \mathrm{M})$, with or without the RAD001 $(1 \mu \mathrm{M})$. After treatment, total protein was extracted from cells, after homogenization in lysis buffer $(10 \mathrm{mM} \mathrm{NaF}, 10$ $\mathrm{mM}$ Na-pyrophosphate, $1 \mathrm{mM} \mathrm{Na} 3 \mathrm{VO} 4$. Invitrogen) containing protease and phosphatase inhibitors (SigmaAldrich). Then the supernatants were obtained using centrifugation at $4{ }^{\circ} \mathrm{C}, 14000$ r.p.m. for $15 \mathrm{~min}$. Total proteins $(50 \mu \mathrm{g})$ were separated using Sodium Dodecyl Sulphate - PolyAcrylamide Gel Electrophoresis (SDSPAGE) at $10 \%$. Proteins on gels were transferred to Nitrocellulose Blotting Membranes $0.45 \mu \mathrm{m}$ (GE Healthcare Life Sciences) for 1.5 hours at $220 \mathrm{~mA}$ in a transfer chamber. Membranes were blocked with 5\% milk in TBST (10 mM Tris-HCl, pH 7.5, $200 \mathrm{mM} \mathrm{NaCl}$, $0.05 \%$ Tween-20) for 1 hour at room temperature and then incubated overnight in primary antibodies at $4{ }^{\circ} \mathrm{C}$. Rabbit monoclonal antibodies for Erk1/2, p-Erk1/2 (Thr202/ Tyr204), S6K1, p-S6K1(Thr389), 4EBP1, p-4EBP1, p-P38 (Thr180/Tyr182), P38, p-Akt (Ser473), Akt were from Cell Signaling Technology. The non-phosphorylated form of each protein was used as internal control. Membranes were washed three times with TBST for 10 minutes and incubated with secondary antibody for 2 hours at room temperature. Secondary antibodies include goat anti-rabbit-HRP (Jackson ImmunoResearch). After 3 TBST washes of 10 minutes each, membranes were revealed through EMD Millipore ImmobilonTM Western Chemiluminescent HRP Substrate (ECL).

\section{Proliferation assay}

BON and NCI-H727 cells were plated into 6-well plates at a density of $50 \times 10^{4}$ cells/well in duplicate. After $24 \mathrm{hrs}, \mathrm{RAD} 001(1 \mu \mathrm{M})$, AMD3100 $(5 \mu \mathrm{M})$ or both agents 
were added. Cells were incubated for 24-48-72 hrs at $37^{\circ} \mathrm{C}$ in a humidified atmosphere containing 5\% CO2 and counted using a hemocytometer.

\section{Statistical analyses}

Statistical analysis was performed using Graphpad 5 software (Graphpad Software, La Jolla, CA, USA) and the results were considered statistically significant at a level of $\mathrm{p}<0.05$. The t Tests, nonparametric Mann-Whitney test, one-tailed was used to analyze relative mRNA expression level of CXCR4/CXCL12/CXCR7 axis in tumors versus non-tumor tissues and it was used to compare the distribution of the markers investigated among the different tumor groups and with clinical and pathological variables. The Spearman's test was used to analyze the correlation index among markers expression. One-way ANOVA and multiple comparisons with Bonferroni correction was used to evaluate the growth cells in vitro.

\section{ACKNOWLEDGMENTS}

This study was in part supported by Multidisciplinary Group for Neuroendocrine Tumours of Naples (ENETS center of excellence for NET)

\section{CONFLICTS OF INTEREST}

The authors declare that there is no conflict of interest that could be perceived as prejudicing the impartiality of the research reported.

\section{REFERENCES}

1. Fraenkel M, Kim M, Faggiano A, de Herder WW, Valk GD, Knowledge N. Incidence of gastroenteropancreatic neuroendocrine tumours: a systematic review of the literature. Endocrine-related cancer. 2014; 21:R153-163.

2. Faggiano A, Ferolla P, Grimaldi F, Campana D, Manzoni M, Davi MV, Bianchi A, Valcavi R, Papini E, Giuffrida D, Ferone D, Fanciulli G, Arnaldi G, Franchi GM, Francia G, Fasola G, et al. Natural history of gastro-entero-pancreatic and thoracic neuroendocrine tumors. Data from a large prospective and retrospective Italian epidemiological study: the NET management study. Journal of endocrinological investigation. 2012; 35:817-823.

3. Karges W, Adler G. [Clinical genetics of neuroendocrine tumors]. Medizinische Klinik. 2003; 98:712-716.

4. Rindi G, Petrone G, Inzani F. The 2010 WHO classification of digestive neuroendocrine neoplasms: a critical appraisal four years after its introduction. Endocrine pathology. 2014; 25:186-192.

5. Pavel M, Baudin E, Couvelard A, Krenning E, Oberg K, Steinmuller T, Anlauf M, Wiedenmann B, Salazar R, Barcelona Consensus Conference p. ENETS Consensus
Guidelines for the management of patients with liver and other distant metastases from neuroendocrine neoplasms of foregut, midgut, hindgut, and unknown primary. Neuroendocrinology. 2012; 95:157-176.

6. Wu HW, Liu YH. [2012 NCCN guideline interpretation of the differentiated thyroid carcinoma]. Zhonghua wai ke za zhi [Chinese journal of surgery]. 2012; 50:675-677.

7. Edge SB, Compton CC. The American Joint Committee on Cancer: the 7th edition of the AJCC cancer staging manual and the future of TNM. Annals of surgical oncology. 2010; 17:1471-1474.

8. Elisei R, Schlumberger MJ, Muller SP, Schoffski P, Brose MS, Shah MH, Licitra L, Jarzab B, Medvedev V, Kreissl MC, Niederle B, Cohen EE, Wirth LJ, Ali H, Hessel $\mathrm{C}$, Yaron $\mathrm{Y}$, et al. Cabozantinib in progressive medullary thyroid cancer. Journal of clinical oncology. 2013; 31:3639-3646.

9. Vainas I, Drimonitis A, Boudina M, Kaprara A, Iakovou I, Salem N, Koussis C. The therapeutic value of SST-A octreotide alone or with adjuvant treatment in patients with advanced medullary thyroid carcinoma and positive (111) In-octreotide scan. Hellenic journal of nuclear medicine. 2005; 8:43-47.

10. Vitale G, Tagliaferri P, Caraglia M, Rampone E, Ciccarelli A, Bianco AR, Abbruzzese A, Lupoli G. Slow release lanreotide in combination with interferon-alpha2b in the treatment of symptomatic advanced medullary thyroid carcinoma. The Journal of clinical endocrinology and metabolism. 2000; 85:983-988.

11. Wells SA, Jr., Robinson BG, Gagel RF, Dralle H, Fagin JA, Santoro M, Baudin E, Elisei R, Jarzab B, Vasselli JR, Read J, Langmuir P, Ryan AJ, Schlumberger MJ. Vandetanib in patients with locally advanced or metastatic medullary thyroid cancer: a randomized, double-blind phase III trial. Journal of clinical oncology. 2012; 30:134-141.

12. Yao JC, Lombard-Bohas C, Baudin E, Kvols LK, Rougier P, Ruszniewski P, Hoosen S, St Peter J, Haas T, Lebwohl D, Van Cutsem E, Kulke MH, Hobday TJ, O'Dorisio TM, Shah MH, Cadiot G, et al. Daily oral everolimus activity in patients with metastatic pancreatic neuroendocrine tumors after failure of cytotoxic chemotherapy: a phase II trial. Journal of clinical oncology. 2010; 28:69-76.

13. Pavel ME, Hainsworth JD, Baudin E, Peeters M, Horsch D, Winkler RE, Klimovsky J, Lebwohl D, Jehl V, Wolin EM, Oberg K, Van Cutsem E, Yao JC, Group R-S. Everolimus plus octreotide long-acting repeatable for the treatment of advanced neuroendocrine tumours associated with carcinoid syndrome (RADIANT-2): a randomised, placebocontrolled, phase 3 study. Lancet. 2011; 378:2005-2012.

14. Yao JC, Shah MH, Ito T, Bohas CL, Wolin EM, Van Cutsem E, Hobday TJ, Okusaka T, Capdevila J, de Vries EG, Tomassetti P, Pavel ME, Hoosen S, Haas T, Lincy J, Lebwohl D, et al. Everolimus for advanced pancreatic neuroendocrine tumors. The New England journal of medicine. $2011 ; 364: 514-523$. 
15. Chiang GG, Abraham RT. Targeting the mTOR signaling network in cancer. Trends Mol Med. 2007; 13:433-442.

16. Cingarlini S, Bonomi M, Corbo V, Scarpa A, Tortora G. Profiling mTOR pathway in neuroendocrine tumors. Targeted oncology. 2012; 7:183-188.

17. Laplante M, Sabatini DM. mTOR signaling in growth control and disease. Cell. 2012; 149:274-293.

18. Faggiano A, Ramundo V, Dicitore A, Castiglioni S, Borghi MO, Severino R, Ferolla P, Crino L, Abbruzzese A, Sperlongano P, Caraglia M, Ferone D, Hofland L, Colao A, Vitale G. Everolimus is an active agent in medullary thyroid cancer: a clinical and in vitro study. Journal of cellular and molecular medicine. 2012; 16:1563-1572.

19. Zitzmann K, De Toni EN, Brand S, Goke B, Meinecke J, Spottl G, Meyer HH, Auernhammer CJ. The novel mTOR inhibitor RAD001 (everolimus) induces antiproliferative effects in human pancreatic neuroendocrine tumor cells. Neuroendocrinology. 2007; 85:54-60.

20. Tamburrino A, Molinolo AA, Salerno P, Chernock RD, Raffeld M, Xi L, Gutkind JS, Moley JF, Wells SA, Jr., Santoro M. Activation of the mTOR pathway in primary medullary thyroid carcinoma and lymph node metastases. Clinical cancer research. 2012; 18:3532-3540.

21. Manfredi GI, Dicitore A, Gaudenzi G, Caraglia M, Persani L, Vitale G. PI3K/Akt/mTOR signaling in medullary thyroid cancer: a promising molecular target for cancer therapy. Endocrine. 2015; 48:363-370.

22. Carew JS, Kelly KR, Nawrocki ST. Mechanisms of mTOR inhibitor resistance in cancer therapy. Targeted oncology. 2011; 6:17-27.

23. Carter Y, Jaskula-Sztul R, Chen H, Mazeh H. Signaling pathways as specific pharmacologic targets for neuroendocrine tumor therapy: RET, PI3K, MEK, growth factors, and Notch. Neuroendocrinology. 2013; 97:57-66.

24. De Dosso S, Grande E, Barriuso J, Castellano D, Tabernero J, Capdevila J. The targeted therapy revolution in neuroendocrine tumors: in search of biomarkers for patient selection and response evaluation. Cancer metastasis reviews. 2013; 32(3-4):465-477.

25. Strosberg J. Evolving treatment strategies for management of carcinoid tumors. Current treatment options in oncology. 2013; 14:374-388.

26. Hashimoto I, Koizumi K, Tatematsu M, Minami T, Cho S, Takeno N, Nakashima A, Sakurai H, Saito S, Tsukada $\mathrm{K}$, Saiki I. Blocking on the CXCR4/mTOR signalling pathway induces the anti-metastatic properties and autophagic cell death in peritoneal disseminated gastric cancer cells. European journal of cancer. 2008; 44:1022-1029.

27. Murooka TT, Rahbar R, Platanias LC, Fish EN. CCL5mediated T-cell chemotaxis involves the initiation of mRNA translation through mTOR/4E-BP1. Blood. 2008; 111:4892-4901.

28. Ierano C, Santagata S, Napolitano M, Guardia F, Grimaldi A, Antignani E, Botti G, Consales C, Riccio A,
Nanayakkara M, Barone MV, Caraglia M, Scala S. CXCR4 and CXCR7 transduce through mTOR in human renal cancer cells. Cell Death Dis. 2014; 5:e1310.

29. Ganju RK, Brubaker SA, Meyer J, Dutt P, Yang Y, Qin S, Newman W, Groopman JE. The alpha-chemokine, stromal cell-derived factor-1alpha, binds to the transmembrane G-protein-coupled CXCR-4 receptor and activates multiple signal transduction pathways. The Journal of biological chemistry. 1998; 273:23169-23175.

30. Muller A, Homey B, Soto H, Ge N, Catron D, Buchanan ME, McClanahan T, Murphy E, Yuan W, Wagner SN, Barrera JL, Mohar A, Verastegui E, Zlotnik A. Involvement of chemokine receptors in breast cancer metastasis. Nature. 2001; 410:50-56.

31. Scala S. Molecular Pathways: Targeting the CXCR4CXCL12 Axis-Untapped Potential in the Tumor Microenvironment. Clinical cancer research. 2015.

32. Burns JM, Summers BC, Wang Y, Melikian A, Berahovich R, Miao Z, Penfold ME, Sunshine MJ, Littman DR, Kuo CJ, Wei K, McMaster BE, Wright K, Howard MC, Schall TJ. A novel chemokine receptor for SDF-1 and I-TAC involved in cell survival, cell adhesion, and tumor development. The Journal of experimental medicine. 2006; 203:2201-2213.

33. Monnier J, Boissan M, L'Helgoualc'h A, Lacombe ML, Turlin B, Zucman-Rossi J, Theret N, Piquet-Pellorce C, Samson M. CXCR7 is up-regulated in human and murine hepatocellular carcinoma and is specifically expressed by endothelial cells. European journal of cancer. 2012; 48:138-148.

34. Zheng K, Li HY, Su XL, Wang XY, Tian T, Li F, Ren GS. Chemokine receptor CXCR7 regulates the invasion, angiogenesis and tumor growth of human hepatocellular carcinoma cells. J Exp Clin Cancer Res. 2010; 29:31.

35. Kaemmerer D, Reimann C, Specht E, Wirtz RM, Sayeg M, Baum RP, Schulz S, Lupp A. Differential expression and prognostic value of the chemokine receptor CXCR4 in bronchopulmonary neuroendocrine neoplasms. Oncotarget. 2015; 6:3346-3358. doi: 10.18632/oncotarget.3242.

36. D'Alterio C, Avallone A, Tatangelo F, Delrio P, Pecori B, Cella L, Pelella A, D'Armiento FP, Carlomagno C, Bianco F, Silvestro L, Pacelli R, Napolitano M, Iaffaioli RV, Scala $\mathrm{S}$. A prognostic model comprising pT stage, $\mathrm{N}$ status, and the chemokine receptors CXCR4 and CXCR7 powerfully predicts outcome in neoadjuvant resistant rectal cancer patients. International journal of cancer. 2014; 135:379-390.

37. Klimstra DS, Modlin IR, Coppola D, Lloyd RV, Suster S. The Pathologic Classification of Neuroendocrine Tumors A Review of Nomenclature, Grading, and Staging Systems. Pancreas. 2010; 39:707-712.

38. Catena L, Bajetta E, Milione M, Ducceschi M, Valente M, Dominoni F, Colonna V. Mammalian target of rapamycin expression in poorly differentiated endocrine carcinoma: clinical and therapeutic future challenges. Targeted oncology. 2011; 6:65-68. 
39. Righi L, Volante M, Rapa I, Tavaglione V, Inzani F, Pelosi G, Papotti M. Mammalian target of rapamycin signaling activation patterns in neuroendocrine tumors of the lung. Endocrine-related cancer. 2010; 17:977-987.

40. Qian ZR, Ter-Minassian M, Chan JA, Imamura Y, Hooshmand SM, Kuchiba A, Morikawa T, Brais LK, Daskalova A, Heafield R, Lin X, Christiani DC, Fuchs CS, Ogino S, Kulke MH. Prognostic significance of MTOR pathway component expression in neuroendocrine tumors. Journal of clinical oncology. 2013; 31:3418-3425.

41. Weekes CD, Song D, Arcaroli J, Wilson LA, RubioViqueira B, Cusatis G, Garrett-Mayer E, Messersmith WA, Winn RA, Hidalgo M. Stromal cell-derived factor 1alpha mediates resistance to $\mathrm{mTOR}$-directed therapy in pancreatic cancer. Neoplasia. 2012; 14:690-701.

42. Munk R, Ghosh P, Ghosh MC, Saito T, Xu M, Carter A, Indig F, Taub DD, Longo DL. Involvement of mTOR in CXCL12 mediated $\mathrm{T}$ cell signaling and migration. PLoS One. 2011; 6:e24667.

43. Portella L, Vitale R, De Luca S, D'Alterio C, Ierano C, Napolitano M, Riccio A, Polimeno MN, Monfregola L, Barbieri A, Luciano A, Ciarmiello A, Arra C, Castello G, Amodeo P, Scala S. Preclinical development of a novel class of CXCR4 antagonist impairing solid tumors growth and metastases. PloS one. 2013; 8:e74548.

44. Tolcher AW, Bendell JC, Papadopoulos KP, Burris HA, 3rd, Patnaik A, Jones SF, Rasco D, Cox DS, Durante M, Bellew KM, Park J, Le NT, Infante JR. A phase IB trial of the oral MEK inhibitor trametinib (GSK1120212) in combination with everolimus in patients with advanced solid tumors. Ann Oncol. 2015; 26:58-64.

45. Niederle MB, Hackl M, Kaserer K, Niederle B. Gastroenteropancreatic neuroendocrine tumours: the current incidence and staging based on the WHO and European Neuroendocrine Tumour Society classification: an analysis based on prospectively collected parameters. Endocr Relat Cancer. 2010; 17:909-918.

46. Komori Y, Yada K, Ohta M, Uchida H, Iwashita Y, Fukuzawa K, Kashima K, Yokoyama S, Inomata M, Kitano S. Mammalian target of rapamycin signaling activation patterns in pancreatic neuroendocrine tumors. Journal of hepato-biliary-pancreatic sciences. 2014; 21:288-295.

47. Klimstra DS. Pathology Reporting of Neuroendocrine Tumors: Essential Elements for Accurate Diagnosis, Classification, and Staging. Semin Oncol. 2013; 40:23-36. 